\title{
Locomotion Imitation of Humanoid Using Goal-directed Self-adjusting Adaptor
}

\author{
Woosung Yang, Nak Young Chong \\ School of Information Science \\ Japan Advanced Institute of Science and Technology \\ Ishikawa, Japan \\ \{woo-yang, nakyoung\}@jaist.ac.jp
}

\author{
ChangHwan Kim, Bum Jae You \\ Intelligent Robotics Research Center \\ Korea Institute of Science and Technology \\ Seoul, Korea \\ \{ckim, ybj\}@kist.re.kr
}

\begin{abstract}
We propose a novel framework for imitation learning that helps a humanoid robot achieve its goal of learning. There are apparent discrepancies in shapes and sizes among humans and humanoid robots. It would be advantageous if robots could learn their behavior from different individuals. Toward this end, this paper discusses appropriate behavior generation method through imitation learning considering that demonstrator and imitator robots have different kinematics and dynamics. As part of a wider interest in behavior generation in general, this work mainly investigates how an imitator robot adapts a reference locomotion gait captured from a demonstrator robot. Specifically, a goal-directed adaptation process that we call self-adjusting adaptor is proposed to achieve stable locomotion of the imitator. The proposed adaptor has an important role that the perceived locomotion patterns are modified to keep the direction of lower leg contacting the ground identical between the demonstrator and the imitator, sustaining the dynamic stability by controlling the position of the center of mass. The validity of the proposed scheme is evaluated through simulations employing various imitator models on OpenHRP and then verified on a real robot.
\end{abstract}

Index Terms - Humanoid robot, Biped locomotion, Imitation learning, Self-adjusting adaptor, ZMP

\section{INTRODUCTION}

Stable and robust dynamic locomotion has been gaining increasing attention in the humanoid robot community. Considerable efforts have been mainly devoted to how to deal with a highly nonlinear nature of robot dynamics and disturbances from an uncertain environment. These efforts include such approaches as the zero moment point criterion [1]-[2], the linear inverted pendulum mode [3], virtual model control [4], and biologically inspired approaches [5]. Even though several methods do not depend heavily on the reference patterns, many existing methods employ some form of pattern generation and tracking control. This requires a fairly accurate model of the robot to compute dynamically admissible patterns. Since humanoid robots have a large number of degrees-of-freedom, efficient pattern generation still remains challenging. Thus, from a practical viewpoint, learning by imitation is considered as a powerful means of enhancing pattern generation competence.

In principle, imitation learning can be classified into the following three cases: what to imitate, how to imitate, and when to imitate [6], [7], [14]. This work falls in the category of how to imitate. Dasgupta and Nakamura proposed a method that enables motion capture data to be transferable to humanoid bipedal locomotion [8]. Many researchers have studied efficient imitation models to obtain reliable motion data in noisy stochastic environments [9]-[13]. Especially, Inamura et al. devised the mimesis model based on HMM which can imitate the motion of others and abstract the timeseries motion patterns as symbol representation [9]. Samejima et al. suggested a special framework MOSAIC, where plural dynamics and inverse dynamics are implemented to predict and control motions [12]-[13]. Also, for human motion generation, Yamane and Nakamura computed the interacting dynamics of structure-varying kinematic chains by incorporating an efficient collision/contact model [15-16].

Although recent progress in imitation learning has yielded notable results in many cases, a unified approach to behavior generation of humanoid robots remains so far largely undetermined. Particularly, only a few approaches have been reported concerning goal-directed imitation that requires effect reproduction through close interaction with the environment. Likewise, even if behaviors can be captured from different individuals, the captured motion data may not be straightforwardly used mainly due to the kinematic and dynamic dissimilarities between the demonstrator and the imitator. For this, we propose a tool for adaptation process that we call "self-adjusting adaptor (SAA)", to promote the ability of the imitator to deal with internal structural constraints as well as external disturbances. What is different from the dynamics filter [16] is that the SAA explicitly incorporates constraints that enable the sustaining of body stability.

In effect, the SAA facilitates imitation mapping between two dissimilar humanoid robots interacting with their environment. This work mainly investigates imitation of locomotion that requires a finely tuned coordination of body segments as well as a stable interaction between the foot and the ground. Specifically, to inspect the significance of robot body dynamics, robots having different sizes and shapes are investigated. The SAA can be used as a framework for goaldirected learning that enables the generation of appropriate behaviors in a variety of different kinds of humanoid robots. The applicability of the proposed method is demonstrated in both simulations using the humanoid robotics software platform, OpenHRP [18], and real robot experiments. 


\section{GOAL-DIRECTED IMITATION LEARNING}

\section{A. Goal-directed imitation between dissimilar bodies}

The difficulties of learning by imitation lie in how to deal with the dissimilar kinematics and dynamics of the demonstrator and the imitator. The main idea of goal-directed imitation proposed in [17] is that the behaviors of the demonstrator and the imitator should bring about the same effect to their environment. To ensure that both robots perform their functions satisfying this condition, the direction of the reaction force at the point of action should be coincident between two bodies. For instance, when learning tennis from a demonstrator having different link lengths, the imitator somehow needs to properly place the racket face vertically against the ball. (See Fig. 1). In the figure, Cases 1 and 2 show that the intended goal might not be achieved if the imitator just copies the trajectories of joint angles or the endeffector of the demonstrator. In contrast, Case 3 illustrates that the imitator modifies the reference trajectories to exert the same effect (the force along the same direction) to the environment (the ball) as the demonstrator performs. Therefore, a good imitator may be capable of adapting the perceived motion trajectories.

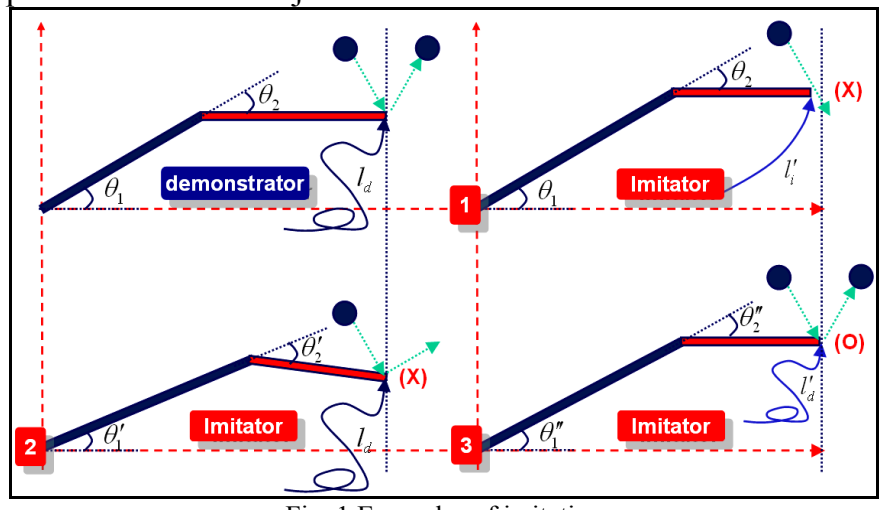

Fig. 1 Examples of imitation

\section{B. Kinematics and dynamics adaptation}

This subsection explains how the perceived reference motions can be regenerated through the SAA. (See Fig. 2.)

1) Phase I: A motion capture system records the trajectories of the link in interaction with the environment and the trajectories of the center of mass (COM) of the demonstrator.

2) Phase II: The captured trajectories are given to the SAA in which the kinematics and dynamics adaptation processes are to be performed. To achieve the intended goal of demonstration from the kinematic point of view, the SAA modifies the input trajectories in the sagittal and frontal planes, separately. When robots interact with their environment, the direction of the link in the interaction should be kept as identical as possible to exert a force in the same direction. The joint angle trajectories of the imitator are regenerated under this condition.

3) Phase III: These joint angle trajectories are controlled through the dynamics adaptation process supported by a neural oscillator network, where the oscillator entrains the sensory feedback data from the environment. This process compensates for the discrepancy of the dynamic properties between the demonstrator and the imitator [17], [19]-[22].

Finally, considering the difference in the number of degrees of freedom and the motion of the individual joints between the demonstrator and the imitator, the demonstrator' motion space is mapped into the imitator's motion space where dynamic stability is guaranteed.

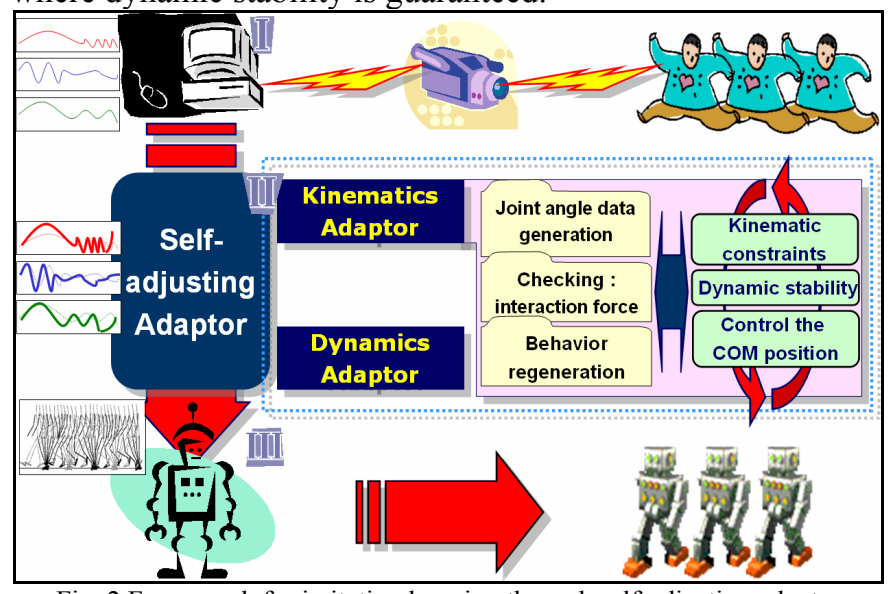

Fig. 2 Framework for imitation learning through self-adjusting adaptor

\section{APPLICATION TO BIPEDAL LOCOMOTION}

In this section, the proposed imitation method is applied to humanoid locomotion imitation illustrated in Fig. 3. For simplicity, we first assume that there are similarities in kinematic configurations between the demonstrator and the imitator. We also assume a uniform mass distribution within the body segments and no limitations of the actuator torque of the imitator. Later, we verify with a kinematically and dynamically dissimilar imitator. The COM is assumed to be located at the hip joint.

At the instance of foot contact against the ground, the height of the COM of imitator is scaled by a ratio of heights between the imitator and the demonstrator and becomes same as the demonstrator's height of the COM. At this moment, the imitator can find its lower leg angle trajectories for the supporting leg to keep the lower leg direction identical with the demonstrator. Then, the ratio of the size of the foot trajectory of the imitator can be determined reflecting the difference between the position on the ground onto which the $\mathrm{COM}$ is projected and the position of the foot in the demonstrator and the imitator. Thus, the imitator's foot trajectory can be generated. Note that this trajectory is obtained solely by a kinematic viewpoint. The imitator learns from the demonstrator about the shape of the foot trajectory, the position of the COM, and the direction of lower leg to imitate the demonstrator's locomotion as closely as possible.

An important underlying assumption behind this idea is that the locomotion data of the demonstrator has been fully optimized and tuned to achieve stable locomotion, keeping the angular momentum constant about the center of mass of the body. Specifically, this means that the direction of the ground reaction or the resultant force acting on the foot passes through the COM of the body. Thus, the imitator keeps the 
direction of lower leg in contact with the ground as well as the position of the COM identical with the demonstrator. This will enable the imitator to maintain stability to a large extent, if the imitator is kinematically and dynamically similar to the demonstrator. More specifically, since the regenerated trajectories are to be optimized by changing the position of the COM to satisfy the ZMP criterion, the imitator's dynamic stability can be maintained irrespective of the differences between the robots. Details are given by the following steps:

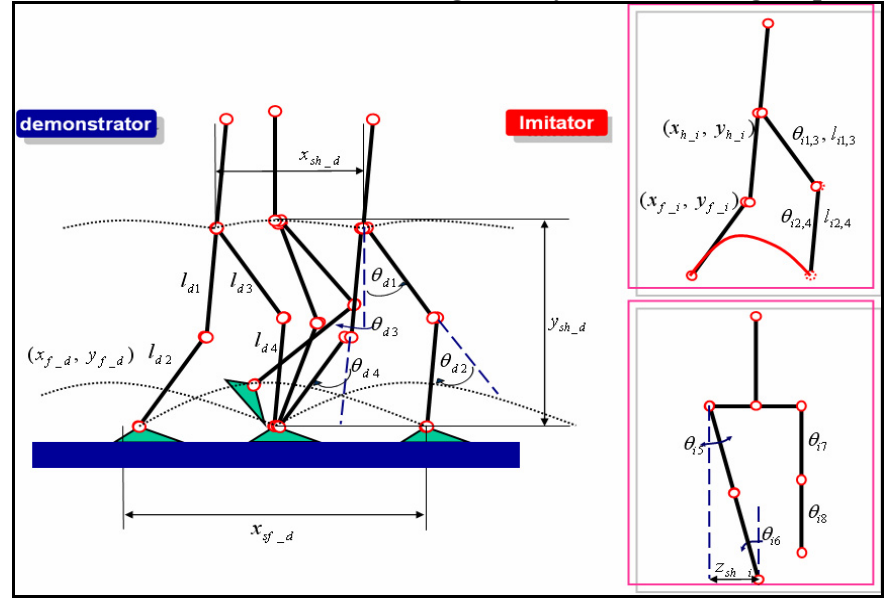

Fig. 3 Trajectories of biped locomotion

$x_{h d}, y_{h d}, x_{f d}, y_{f d}$, and $z_{h d}$ are hip (or waist) and foot (or ankle) trajectories of demonstrator in Cartesian coordinates acquired by such a sensor as a vision camera. $x_{s h_{-} d}, y_{s h_{-} d}, x_{s f_{-} d}$, $y_{s f_{-} d}$ and $z_{s h_{-} d}$ are the one stride interval of hip and foot of demonstrator, respectively. $l_{d 1}$ and $l_{d 2}$ indicate the lengths of the upper and lower limb of the swing leg of demonstrator as seen in Fig. 3. $l_{d 3}$ and $l_{d 4}$ are the lengths of the supporting leg in the figure. Note that the subscription, ' $d$ ', in those lengths denotes the demonstrator and the subscript ' $i$ ' is used for the lengths of the imitator in the figure and the entire paper. The locomotion trajectories are switched between the two legs consecutively in the entire period of locomotion.

1) Step 1: Obtain the trajectories of the hip and the foot of demonstrator in Cartesian coordinates from the motion capturing process: $x_{h_{-} d}, y_{h_{-} d}, x_{f_{-} d}, y_{f_{-} d}$, and $z_{h_{-} d}$ are given.

2) Step 2: Determine the joint angle data for the demonstrator by solving the inverse kinematics problem. In the sagittal plane, $\theta_{d 1}, \theta_{d 2}, \theta_{d 3}$, and $\theta_{d 4}$ are calculated as follows:

$\theta_{d 1,3}=\pi / 2+\tan ^{-1}\left(y_{f, h_{-} d} / x_{f, h_{-} d}\right)-\tan ^{-1}\left(\alpha /\left(k+l_{d 1,3}^{2}-l_{d 2,4}^{2}\right)\right.$,

$\theta_{d 2,4}=\tan ^{-1}\left(\alpha /\left(k-l_{d 1,3}^{2}-l_{d 2,4}^{2}\right)\right)$,

where $\alpha=\sqrt{\left(\left(k+l_{d 1,3}^{2}+l_{d 2,4}^{2}\right)^{2}-2\left(k^{2}+l_{d 1,3}^{4}+l_{d 2,4}^{4}\right)\right.}, k=x_{f, h_{-} d}^{2}+y_{f, h_{-} d}^{2}$. In the same manner, $\theta_{d 5}$ and $\theta_{d \sigma}$ can be obtained by Eq. (1) replacing $x_{f, h_{-} d}$ with $z_{h_{-} d}$. Note that the first row in Eq. (1) contains two equations for $\theta_{d l}$ and $\theta_{d 3}$ such that the first element of the subscript of every variable on the right-hand side of the equation corresponds to $\theta_{d l}$ and the second element $\theta_{d 3}$. The same notation is applied to entire equations.

3) Step 3: Scale down (or up) the link length of demonstrator so that demonstrator and imitator have identical overall height and regenerate foot and hip trajectories: $x_{h_{-}} d$, $y_{, h_{-} d}^{\prime}, x_{f_{-} d}^{\prime}, y_{f_{-} d}^{\prime}$, and $z_{h_{-} d}$

$y_{f_{-} d}^{\prime-d}=l_{d 1}^{-} \rho_{f} \cos \theta_{d 1}+l_{d 2}^{-} \rho_{f} \cos \left(\theta_{d 1}+\theta_{d 2}\right)$

$x_{f_{-} d}^{\prime}=l_{d 1} \rho_{f} \sin \theta_{d 1}+l_{d 2} \rho_{f} \sin \left(\theta_{d 1}+\theta_{d 2}\right)$

$y_{h_{-} d}^{\prime}=l_{d 3} \rho_{h} \cos \theta_{d 3}+l_{d 4} \rho_{h} \cos \left(\theta_{d 3}+\theta_{d 4}\right)$

$x_{h_{-} d}^{\prime}=l_{d 3} \rho_{h} \sin \theta_{d 3}+l_{d 4} \rho_{h} \sin \left(\theta_{d 3}+\theta_{d 4}\right)$

$z_{h_{-} d}^{\prime}=l_{d 3} \rho_{h} \sin \theta_{d 3}+l_{d 4} \rho_{h} \sin \left(\theta_{d 3}+\theta_{d 4}\right)$,

where $\rho_{f}=\frac{l_{i 1}+l_{i 2}}{l_{d 1}+l_{d 2}}, \rho_{h}=\frac{l_{i 3}+l_{i 4}}{l_{d 3}+l_{d 4}}$

4) Step 4: Keep the direction of lower leg under contact status to yield a posture of a behavior goal. Then, the joint angles of imitator are written as follows:

$\theta_{i 1,3}=\cos ^{-1}\left\{\frac{l_{d 1,3} \rho_{f, h} \cos \theta_{d 1,3}+\left(l_{d 2,4} \rho_{f, h}-l_{i 2,4}\right) \cos \left(\theta_{d 1,3}+\theta_{d 2,4}\right)}{l_{i 1,3}}\right\}$

$\theta_{i 2,4}=\left(\theta_{d 1,3}+\theta_{d 2,4}\right)-\theta_{i 1,3}$,

where $\rho_{f, h}=\left(l_{i 1,3}+l_{i 2,4}\right) /\left(l_{d 1,3}+l_{d 2,4}\right)$ and $\left(\theta_{d 1,3}+\theta_{d 2,4}\right)$ mean the direction of end link of the demonstrator. This procedure is performed when the foot of demonstrator is fully in contact with the ground. In this paper, it is assumed that the foot contact is modeled simply as a point contact at the ankle joint.

5) Step 5: Generate foot and hip trajectories of imitator, multiplying the constant of ratios defined below to the scaled trajectories of foot and hip of demonstrator obtained from Step 3 as

$$
\begin{aligned}
& y_{f_{-} i}=\text { ratio }_{y_{-} f} y_{f_{-} d}^{\prime}, x_{f_{-} i}=\text { ratio }_{x_{-} f} x_{f_{-} d}^{\prime}, y_{h_{-} i}=\text { ratio }_{y_{-} h} y_{h_{-}}^{\prime} \\
& x_{h_{-} i}=\text { ratio }_{x_{-} h} x_{h_{-} d}^{\prime}, z_{h_{-} i}=\text { ratio }_{z_{-} h} z_{h_{-} d}^{\prime},
\end{aligned}
$$

where

$$
\begin{aligned}
\text { ratio }_{x_{-} f, h} & \left|1-\frac{x_{f, h_{-} i}}{x_{f, h_{-} d}}\right|, \text { ratio }_{y_{-} f, h}=\left|1-\frac{y_{f, h_{-} i}}{y_{f, h_{-} d}}\right|, \text { ratio }_{z_{-} h}=\left|1-\frac{y_{h_{-} i}}{y_{h_{-} d}}\right| \\
x_{f, h_{-} i}= & l_{d 1,3} \rho_{f, h} \sin \theta_{d 1,3}+l_{d 2,4} \rho_{f, h} \sin \left(\theta_{d 1,3}+\theta_{d 2,4}\right) \\
& -\left(l_{i 1,3} \sin \theta_{i 1,3}+l_{i 2,4} \sin \left(\theta_{i 1,3}+\theta_{i 2,4}\right)\right) \\
y_{f, h_{-} i}= & l_{d 1,3} \rho_{f, h} \cos \theta_{d 1,3}+l_{d 2,4} \rho_{f, h} \cos \left(\theta_{d 1,3}+\theta_{d 2,4}\right) \\
& -\left(l_{i 1,3} \cos \theta_{i 1,3}+l_{i 2,4} \cos \left(\theta_{i 1,3}+\theta_{i 2,4}\right)\right) \\
z_{h_{-} i}= & l_{d 3} \rho_{h} \sin \theta_{d 3}+l_{d 4} \rho_{h} \sin \left(\theta_{d 3}+\theta_{d 4}\right) \\
& -\left(l_{i 3} \sin \theta_{i 3}+l_{i 4} \sin \left(\theta_{i 3}+\theta_{i 4}\right)\right)
\end{aligned}
$$

6) Step 6: Finally, acquire the joint angle of imitator by inverse kinematics, which is basically the same as Eq. (1), and judge whether the obtained values are reasonable or not in kinematic and dynamic sense: $\theta_{i 1}, \theta_{i 2}, \theta_{i 3}, \theta_{i 4}, \theta_{i 5}$, and $\theta_{i 6}$

7) Step 7: If the kinematic constraint or stability problem arises, perform the optimization process to control the waist position of imitator as follows. During the optimization, the position of the waist of imitator is rearranged satisfying two constraints. At first, the right foot and left foot should be in contact with the ground in the double support phase. At the same time, in the rearranged position of the waist, the regenerated locomotion trajectory should satisfy the ZMP criterion. This problem is formulated separately in the sagittal and frontal planes. Specifically, in the sagittal plane, the 
minimal adjusting amount of the hip joint's trajectories is determined satisfying such two constraints as two foots on the ground and the ZMP criterion. This minimization problem can be given as follows,

subject to

$$
\min _{\Delta \theta_{i 1}} f\left(\Delta \theta_{i 1}\right)=\left|y_{f_{-} i}\left(\theta_{i 1}+\Delta \theta_{i 1}\right)-y_{d_{-} i}^{\prime}\right|_{y}
$$

$$
\begin{aligned}
& \text { i) } y_{R}\left(\theta_{i 1}+\Delta \theta_{i 1}\right)=0, y_{L}\left(\theta_{i 1}+\Delta \theta_{i 1}\right)=0 \\
& \text { ii) } \varepsilon_{x} \leq x_{z m p}\left(\theta_{i 1}+\Delta \theta_{i 1}\right) \leq \delta_{x},
\end{aligned}
$$

where $y_{R}$ and $y_{L}$ are the distance between ground and foot position at right leg and left leg, respectively. A given $\varepsilon_{x}$ and $\delta_{x}$ are the ZMP limitation of imitator's foot in the $x$ direction in this paper. And

$$
\begin{aligned}
x_{z m p} & =\frac{\sum_{n} m_{n}\left(\ddot{y}_{n}+g\right) x_{n}-\sum_{n} m_{n} \ddot{x}_{n} y_{n}-\sum_{n} I_{n z} \ddot{z}_{i z}}{\sum_{n} m_{n}\left(\ddot{y}_{n}+g\right)} \\
z_{z m p} & =\frac{\sum_{n} m_{n}\left(\ddot{y}_{n}+g\right) z_{n}-\sum_{n} m_{n} \ddot{z}_{n} y_{n}-\sum_{n} I_{i x} \ddot{\theta}_{i x}}{\sum_{n} m_{n}\left(\ddot{z}_{n}+g\right)},
\end{aligned}
$$

where $y_{n}=l_{n} \cos \theta_{n}, x_{n}=l_{n} \sin \theta_{n}, z_{n}=l_{n} \sin \theta_{n}$ for $n=1 \sim 6$.

Similarly, the minimization problem in the frontal plane can be formulated as

subject to

$$
\min _{\Delta \theta_{i 5}} f\left(\Delta \theta_{i 5}\right)=\left|z_{h_{-} i}\left(\theta_{i 5}+\Delta \theta_{i 5}\right)-z_{h_{-} d}^{\prime}\right|_{z}
$$

$$
\text { i) } z_{L}\left(\theta_{i 5}+\Delta \theta_{i 5}\right)=0
$$

ii) $\varepsilon_{z} \leq z_{z m p}\left(\theta_{i 5}+\Delta \theta_{i 5}\right) \leq \delta_{z}$,

where $z_{L}$ is the distance between the maximum displacement of the COM in the desired and modified trajectories. $\mathcal{E}_{z}$ and $\delta_{z}$ are the ZMP limitation of imitator's foot in the $z$ direction. The above optimization process can be performed at each time interval, $t=k T$ for $k=1 \sim n$, in the entire period of locomotion. $T$ denotes one stride interval. This enables the imitator to acquire the upper leg angle trajectories offering a dynamically stable COM height during locomotion. In the problem, the COM position is controlled by $\Delta \theta_{i 1}$ on which $\theta_{i 2}, \theta_{i 3}$, and $\theta_{i 4}$ are dependent. Also, $\theta_{i 6}$ is dependent on $\Delta \theta_{i 5}$. Therefore, if the imitator's foot and hip trajectories are obtained, which are the best possible trajectories with stability limits, the required joint angles of each leg for the imitator can be calculated by solving the inverse kinematics problem as mentioned in Step 2.

\section{SimUlation RESULTS AND DISCUSSION}

We performed extensive simulations to evaluate the SAA using OpenHRP. A built-in test model in OpenHRP is employed as the demonstrator. For different imitators, the test model with variable link lengths and an off-the-shelf humanoid robot model are employed as listed in Table I. The demonstrator and imitators in Cases 1 through 4 have the same total leg length that is the sum of the lengths of upper and lower leg. However those models have different length ratios between the upper and lower leg for each case. In Cases 5 through 9 , we test the imitators having different total length. The model in Case 9 shows differences in the number of degrees of freedom, the kinematic structures, and the dynamic properties including the COM position, the mass and moment of inertia for each link, and the torque limit at each joint. Fig.
5 is a reference motion data obtained by virtually capturing the locomotion trajectories of the demonstrator. The upper and lower lines are the $\mathrm{x}$ - and $\mathrm{y}$-trajectories of one gait cycle in time, respectively, in Cartesian coordinates. They represent

\begin{tabular}{|c|c|c|c|c|c|c|}
\hline & $\begin{array}{c}\text { Total } \\
\text { Height }\end{array}$ & $\begin{array}{l}\text { Case } \\
\text { No. }\end{array}$ & \begin{tabular}{|c|} 
Upper \\
Leg[m]
\end{tabular} & \begin{tabular}{|l|} 
Lower \\
Leg[m]
\end{tabular} & $\begin{array}{l}\text { Kinematic } \\
\text { structure }\end{array}$ & $\begin{array}{l}\text { Dynamic } \\
\text { property }\end{array}$ \\
\hline \multirow{8}{*}{ Imitator } & \multirow{2}{*}{ Same } & 1 & $\begin{array}{c}0.3435 \\
(2.83 \%) \\
\end{array}$ & $\begin{array}{c}0.31 \\
(3.33 \%) \\
\end{array}$ & Same & Same \\
\hline & & 2 & $\begin{array}{c}0.3635 \\
(2.83 \%) \\
\end{array}$ & $\begin{array}{c}0.29 \\
(3.33 \%) \\
\end{array}$ & Same & Same \\
\hline & \multirow{2}{*}{ Same } & 3 & \begin{tabular}{|c|}
0.3035 \\
$(14.1 \%)$ \\
\end{tabular} & \begin{tabular}{|c|}
0.25 \\
$(16.7 \%)$ \\
\end{tabular} & Same & Same \\
\hline & & 4 & \begin{tabular}{|c|}
0.4035 \\
$(14.1 \%)$ \\
\end{tabular} & \begin{tabular}{|c|}
0.35 \\
$(16.7 \%)$ \\
\end{tabular} & Same & Same \\
\hline & \multirow{2}{*}{0.8 times } & 5 & \begin{tabular}{|c|}
0.2728 \\
$(22.8 \%)$ \\
\end{tabular} & $\begin{array}{c}0.25 \\
(16.7 \%) \\
\end{array}$ & Same & Same \\
\hline & & 6 & $\begin{array}{c}0.2928 \\
(17.2 \%) \\
\end{array}$ & $\begin{array}{c}0.23 \\
(23.3 \%) \\
\end{array}$ & Same & Same \\
\hline & \multirow{2}{*}{1.2 times } & 7 & $\begin{array}{c}0.4142 \\
(17.2 \%) \\
\end{array}$ & $\begin{array}{c}0.37 \\
(23.3 \%) \\
\end{array}$ & Same & Same \\
\hline & & 8 & $\begin{array}{c}0.4342 \\
(17.2 \%) \\
\end{array}$ & $\begin{array}{c}0.35 \\
(23.3 \%) \\
\end{array}$ & Same & Same \\
\hline $\begin{array}{c}\text { Imitator } \\
\text { (HOAP-1) }\end{array}$ & Different & 9 & \begin{tabular}{|c|}
0.1 \\
$(28.3 \%)$ \\
\end{tabular} & $\begin{array}{c}0.1 \\
(33.4 \%) \\
\end{array}$ & Different & Different \\
\hline Demonstrator & & & 0.3535 & 0.3 & & \\
\hline
\end{tabular}
the foot and hip trajectories of the demonstrator in swing phase of the left and right leg in turn.

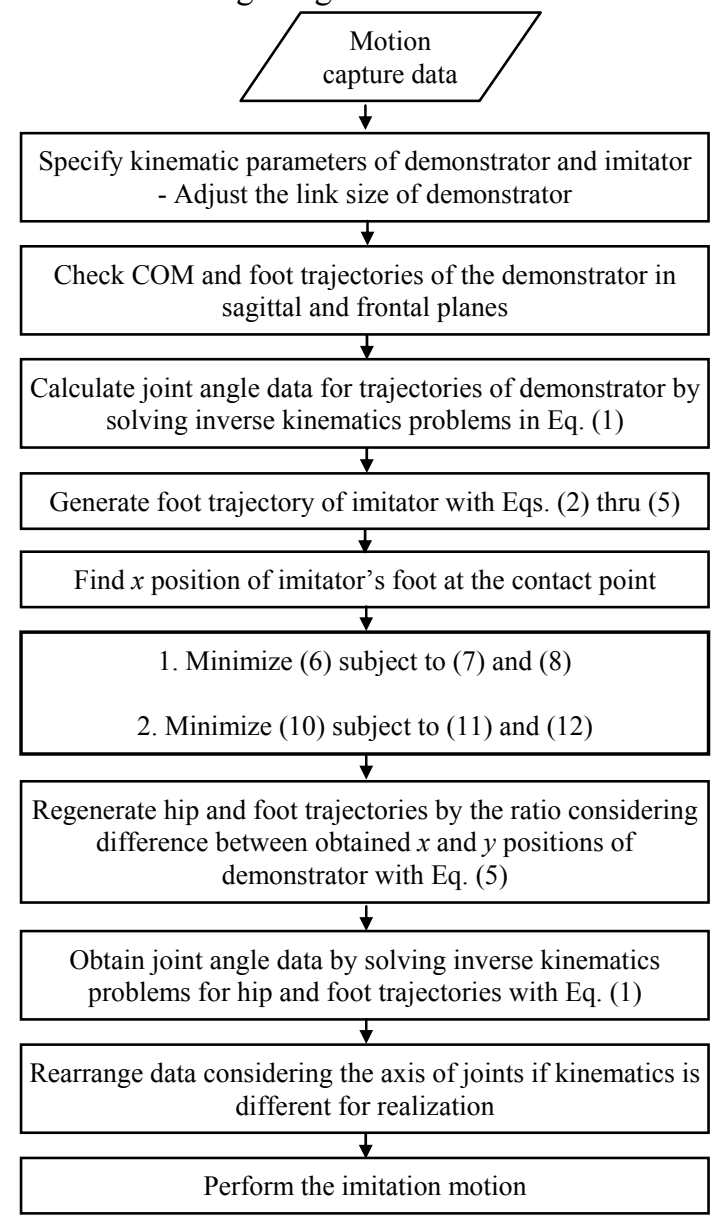

Fig. 4 Flowchart of SAA for imitator pattern generation TABLE I

SPECIFICATIONS OF EMPLOYED MODELS 
Table I shows the height and limb length difference between various imitator models with respect to the common demonstrator. In the kinematics adaptation process, we need to pay attention to the problems in which the kinematic constraints are violated. Most cases are observed either when the leg joint angles satisfying Eqs. (7) and (11) at the moment of heel strike might not exist, or when the leg joint trajectories of one stride (connecting heel strike to heel strike) might not exist. These problems mainly occur when the length of the imitator's upper leg is shorter than the demonstrator's upper leg, that is, the generated area of the foot trajectory geometry for the imitator is larger than that for the demonstrator. To overcome these difficulties, we modified the height of the COM of the imitator to satisfy the ZMP criterion. For cases 3 , 5,7 and 9 the modification of the height of COM was performed, while there were no violations on the kinematic constraints for the cases 1, 2, 4, 6 and 8 .

There are significant dissimilarities in the limb length ratio in Case 7 and differences in dynamic properties and kinematic structure in Case 9. Figs 6 and 7 are the hip and knee joint angle trajectories of the imitator regenerated using Eq. (4). In these figures, the accordance of the middle graph at both ends (encircled with black dotted lines) implies that the direction of the lower leg of the demonstrator and the imitator is kept identical at the moment of heel strike. It can be verified from this simulation that the foot trajectory of the regenerated imitator's foot is similar to that of the original demonstrator's foot. As expected, the regenerated stride interval of the imitator is longer than that of the demonstrator in both cases of 7 and 9. To prevent the imitator from violating the kinematic constraints, the imitator's COM height needs to be modified through the optimization process. In contrast, we can observe that the original joint angle trajectories of the demonstrator are inadmissible for the imitators, since it causes abnormally large ground reaction forces and tilted trajectories. Therefore, the dynamic balance of the imitator motion can not be obtained.

Finally, we simulate the biped locomotion of the imitator on the OpenHRP employing the PD controller and the HighGain controller. One model is actuated by the input data for the demonstrator and the other model is actuated by the input data modified by the SAA. Figs. 8 and 9 represent the ankle joint's relative force, relative torque, and actuating torque of the imitator when the SAA is incorporated and not incorporated, respectively. It is evident that the locomotion becomes unfeasible in the white dashed line area in Fig 8, while the rhythmic patterns are maintained in Fig. 9. Figures 10 to 13 are the snapshots of OpenHRP animation. In Figs. 10 and 12 , the imitators actuated by the original demonstrator data fall down and the imitators actuated by the modified data with the SAA walk stably as shown in Figs 11 and 13. Fig. 14 shows snapshots of a real robot walking facilitated by the proposed method.
This paper so far discussed the kinematics adaptation process of the SAA. To realize a more functionally robust SAA, we need to consider an efficient way of dealing with the dynamic characteristics of the demonstrator and the imitator. In practice, it is required to estimate the mass distribution within the body segments, in addition to the length of the segments, of the demonstrator. Instead of tackling this problem directly, we have attempted to minimize the difference in body dynamics between the demonstrator and the imitator by changing the imitator's height of the COM reflecting the ZMP criterion. From the simulation results of Case 9, it was verified that the SAA compensated, to a large extent, for the differences of dynamics. Thus, we can obtain dynamically admissible trajectories for the imitator. Most of the remaining problems associated with unknown terrain as well as additional uncertainty in dynamic characteristics can be compensated by exploiting a neural oscillator network. The neural oscillator is known to be robust to changes in dynamic parameters or environment disturbances over a specific range of frequencies. This will be addressed in future phases of this work.

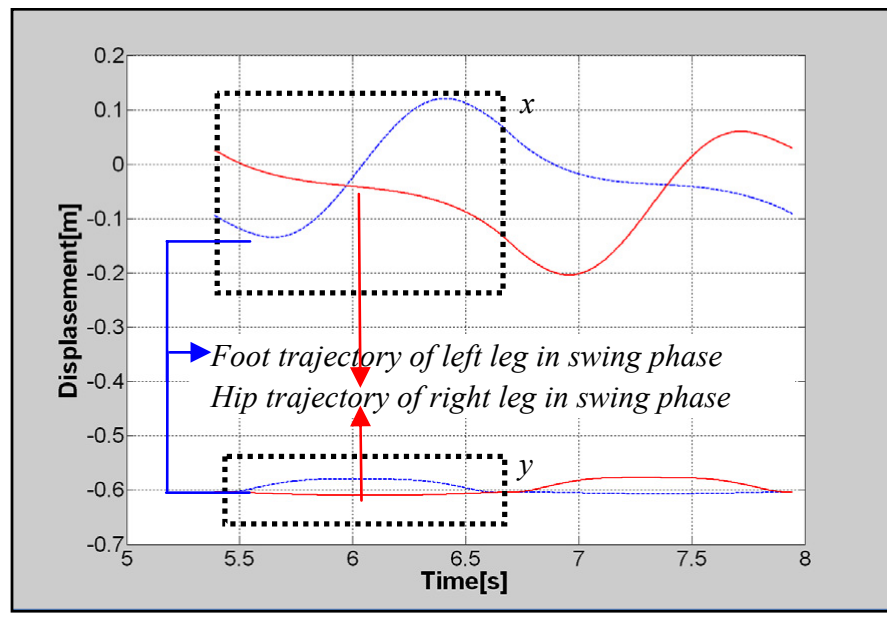

Fig. 5 Foot and hip trajectories of the demonstrator in $x$ - and $y$-coordinates

\section{A. $\quad$ Case 7}

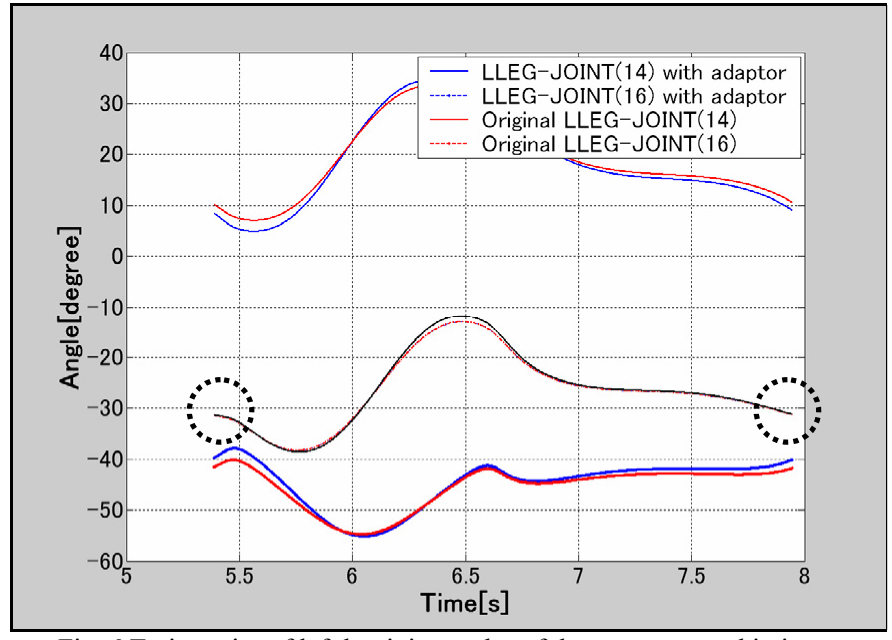

Fig. 6 Trajectories of left leg-joint angles of demonstrator and imitator 


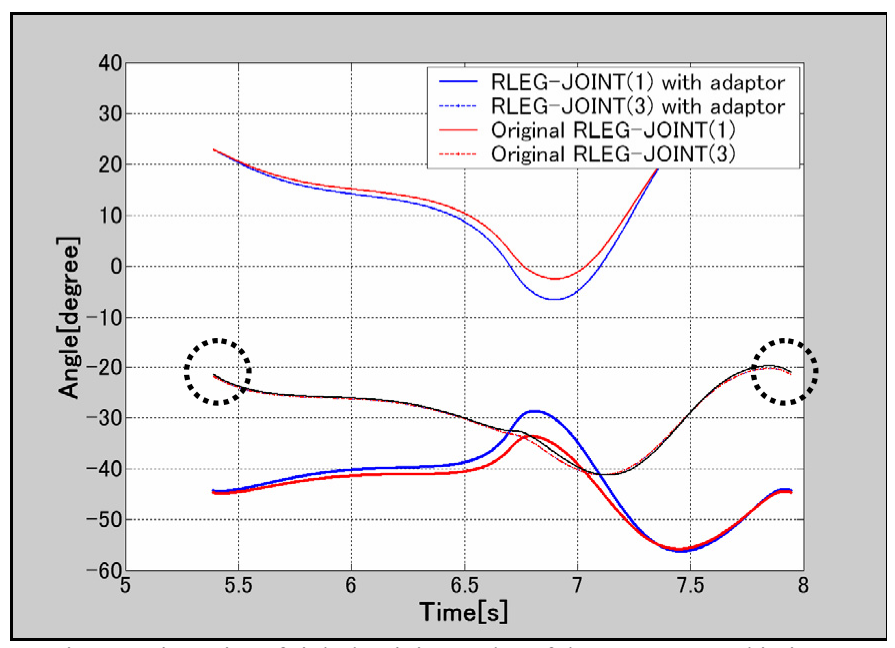

Fig. 7 Trajectories of right leg-joint angles of demonstrator and imitator

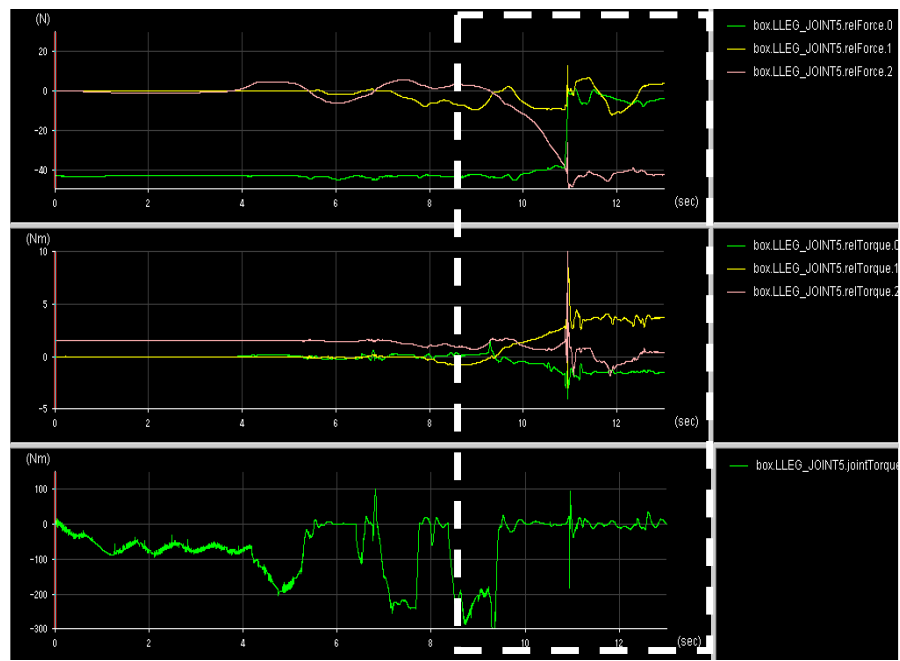

Fig. 8 Relative force, torque, and actuating torque at ankle joint without SAA

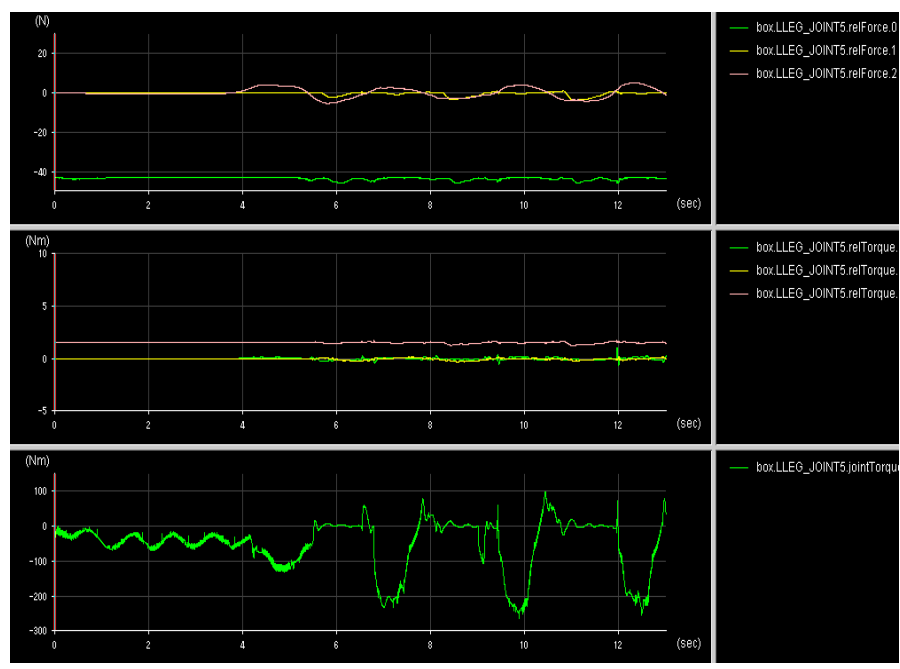

Fig. 9 Relative force, torque, and actuating torque at ankle joint with SAA

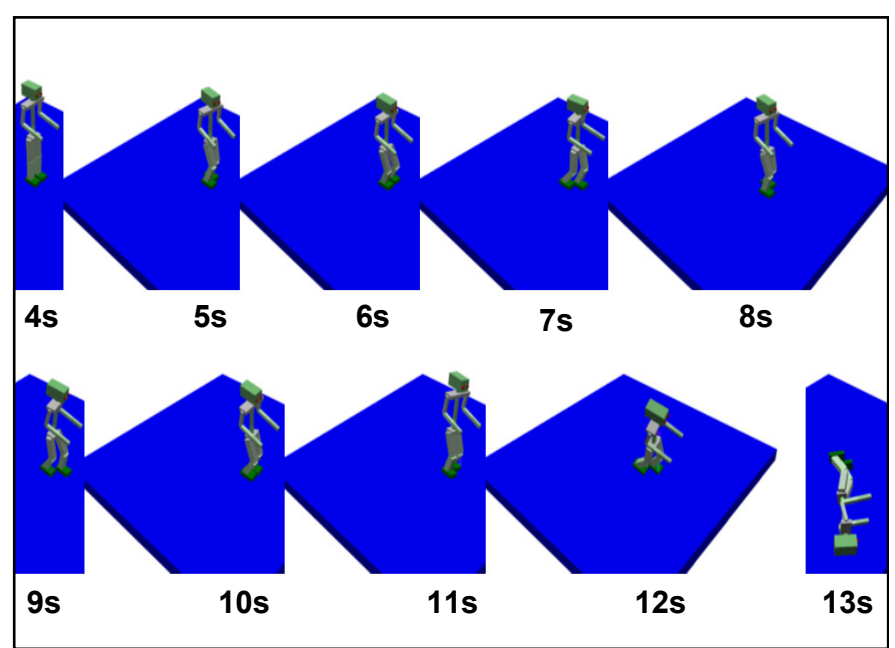

Fig. 10 Snapshots of imitator's locomotion without SAA

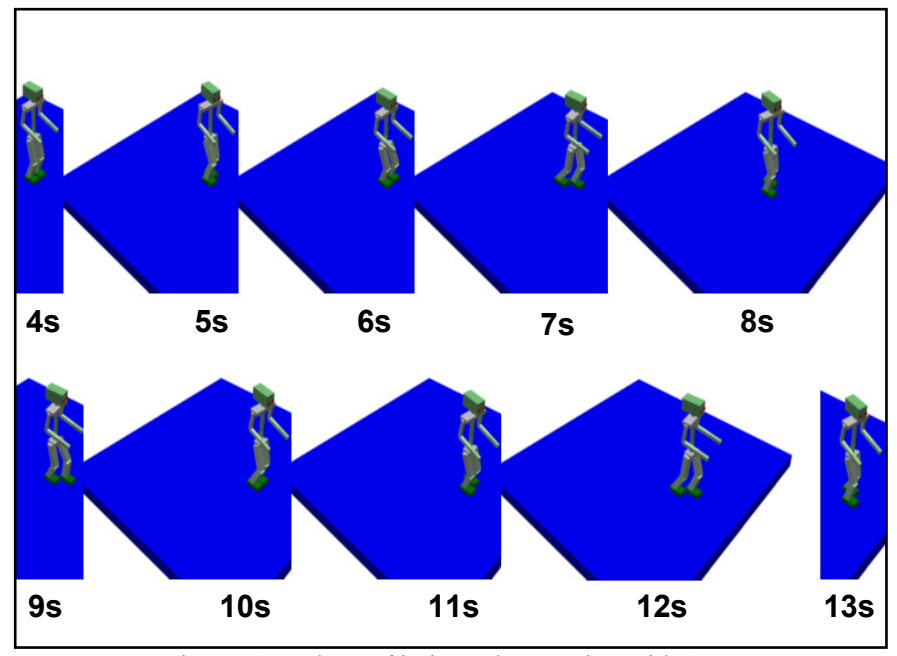

Fig. 11 Snapshots of imitator locomotion with SAA

B. Case 9

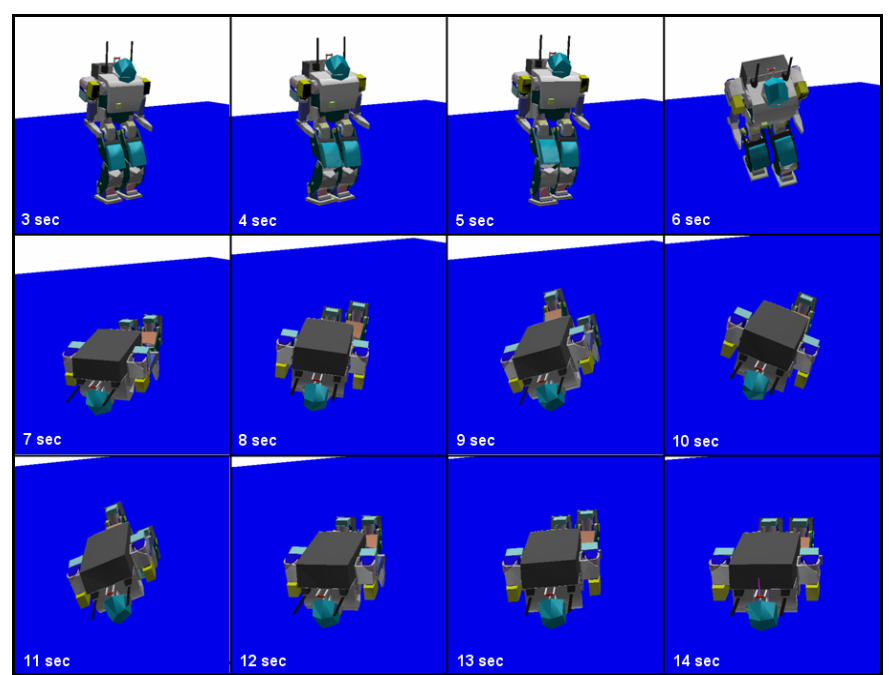

Fig. 12 Snapshots of imitator locomotion without SAA 


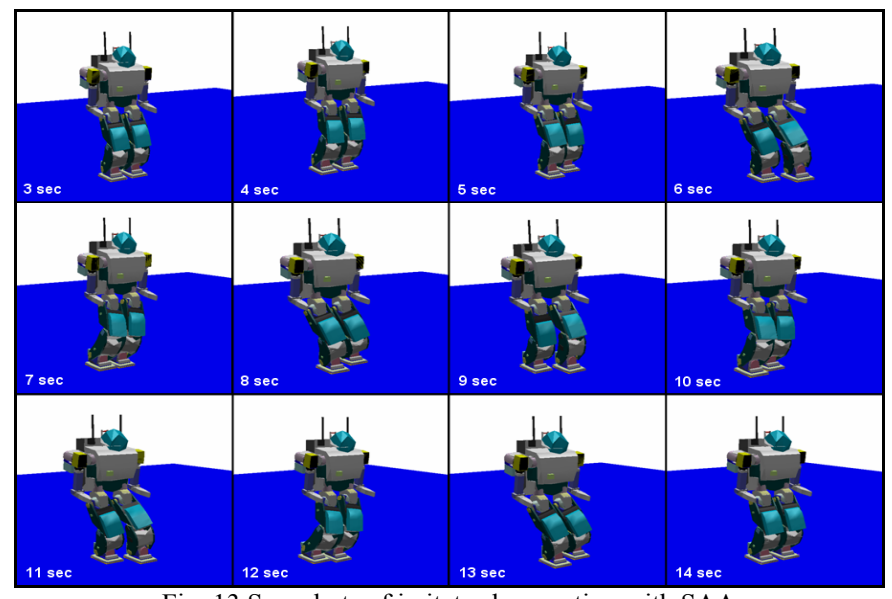

Fig. 13 Snapshots of imitator locomotion with SAA

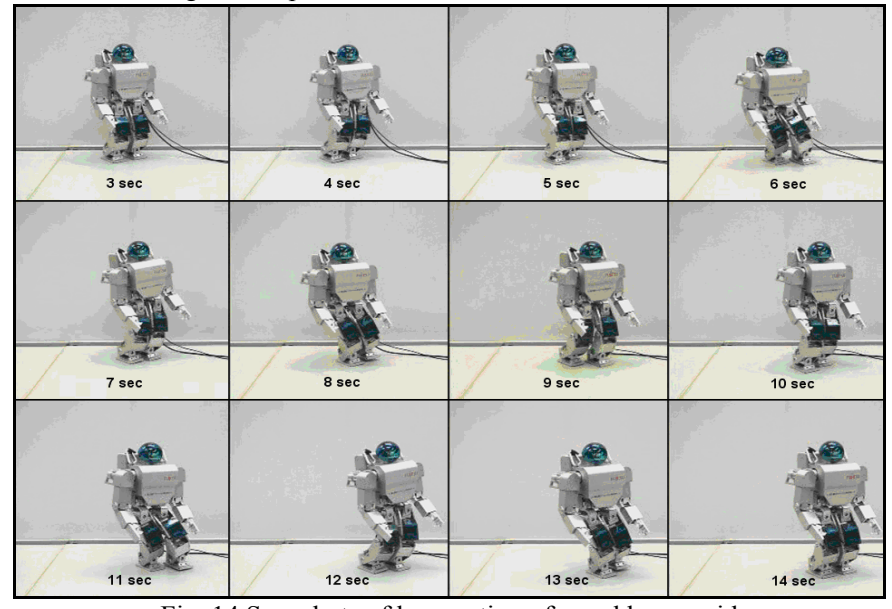

Fig. 14 Snapshots of locomotion of a real humanoid

\section{CONCLUSION}

In this paper, a new framework for imitation was presented to enable a humanoid robot to learn new behaviors from other robots. No works clearly explained how imitation techniques could be incorporated into learning that guarantees the achievement of the intended goal of demonstration such as bipedal locomotion. This work led to the formulation and verification of a practical approach to the real-world learning by imitation. Our major contributions can be summarized as: 1) this work specifically investigated the case when the robot was closely interacted with the environment. 2) this approach allowed for learning from other robots in different shapes and bodies. To implement these, a practical methodology called self-adjusting adaptor was developed. The proposed adaptor was verified by locomotion learning on the virtual humanoid robot simulator OpenHRP. Using a reference locomotion pattern originally generated for the test model in OpenHRP, the patterns suitable to nine different humanoid models were adjusted. From the simulation results, it was observed that the proposed adaptor was of use for humanoid walking. In addition, we verified this proposed adaptor through experiments with a real robot. We will apply the adaptor to the learning of various motions in future work. Finally, we will include a neural oscillator network which can compensate for unknown dynamic properties and external disturbances.

\section{ACKNOWLEDGMENT}

This research is conducted as a program for the "Fostering Talent in Emergent Research Fields" in Special Coordination Funds for Promoting Science and Technology by Ministry of Education, Culture, Sports, Science and Technology of Japan.

\section{REFERENCES}

[1] M. Vukobratovic and D. Juricic, "Contribution to the Synthesis of Biped Gait," IEEE Trans. Bio-Med. Eng., vol. BME-16, no.1, pp. 1-6 (1969)

[2] M. Vukobratovic, "How to Control Artificial Anthropomorphic Systems," IEEE Trans. Syst., Man, Cybern., vol. SMC-3, no.5, pp. 497-507 (1973)

[3] S. Kajita, O. Matsumoto, and M. Saigo, "Real-time 3D Walking Pattern Generation for a Biped Robot with Telescopic Legs," Proc. IEEE Int. Conf. on Robotics and Automation, pp. 2299-2306 (2001)

[4] J. Pratt, C.-M. Chew, A. Torres, P. Dilworth, and G. Pratt, "Virtual Model Control: An Intuitive Approach for Biped Locomotion," The Int. Journal of Robotics Research, vol. 20, no. 2, pp. 129-143 (2001)

[5] G. Endo, J. Nakanishi, J. Morimoto, and G. Cheng, "Experimental Studies of a Neural Oscillator for Biped Locomotion with QRIO," IEEE Int. Conf. on Robotics and Automation, pp. 598-604 (2005)

[6] C. Nehaniv and K. Dautenhahn, "Of Hummingbirds and Helicopters: An Algebraic Framework for Interdisciplinary Studies of Imitation and Its Applications," An Interdisciplinary Approaches to Robot Learning, J. Demiris and A. Birk (Eds.), World Scientific Publishing Co., pp. 136-161 (2000)

[7] A. Billard and R. Siegwart, "Robot Learning from Demonstration," Robotics and Autonomous Systems, vol. 47, pp. 65-67 (2004)

[8] A. Dasgupta and Y. Nakamura, "Making Feasible Walking Motion of Humanoid Robots from Human Motion Capture Data," Proc. IEEE Int. Conf. on Robotics and Automation, pp. 1044-1049 (1999)

[9] T. Inamura, I. Toshima, H. Tanie, and Y. Nakamura, "Embodied Symbol Emergence Based on Mimesis Theory," The Int. Journal of Robotics Research, vol. 23, no. 4-5, pp. 363-377 (2004)

[10] A. J. Ijspeert, J. Nakanishi, and S. Schaal, "Movement Imitation with Nonlinear Dynamical Systems in Humanoid Robots," Proc. IEEE Int. Conf. on Robotics and Automation, pp. 1398-1403 (2002)

[11] S. Schaal, "Is Imitation Learning the Way to Humanoid Robots?," Trends in Cognitive Science, vol. 3, no. 6, pp. 233-242 (1999)

[12] K. Samejima, K. Katagiri, K. Doya, and M. Kawato, "Symbolization and Imitation Learning of Motion Sequence Using Competitive Modules," Trans. of the Institute of Electronics, Information, and Communication Engineers, vol. J85-D-II, no. 1, pp. 90-100 (2002)

[13] K. Samejima, K. Doya, and M. Kawato, "Inter-module Credit Assignment in Modular Reinforcement Learning," Neural Networks, vol. 16, no. 7, pp. 985-994 (2003)

[14] J. Demiris and G. Hayes, "Do Robots Ape?," Proc. AAAI Fall Symposium on Socially Intelligent Agents, pp. 28-30 (1997)

[15] Y. Nakamura and K. Yamane, "Dynamics Computation of Structurevarying Kinematic Chains and Its Application to Human Figures," IEEE Trans. on Robotics and Automation, vol. 16, no. 2, pp. 124-134 (2000)

[16] K. Yamane and Y. Nakamura, "Dynamics Filter - Concept and Implementation of Online Motion Generator for Human Figures," IEEE Trans. on Robotics and Automation, vol. 19, no. 3, pp. 421-432 (2003)

[17] W. Yang and N. Y. Chong, "Goal-directed Imitation with Self-adjusting Adaptor Based on a Neural Oscillator Network," Proc. Int. Conf. on Advanced Robotics, pp. 404-410 (2005)

[18] http://www.is.aist.go.jp/humanoid/openhrp/English/indexE.html

[19] W. Yang and N. Y. Chong, "Dynamic Systems Control Using Entrainment-enhanced Neural Oscillator," Proc. Int. Conf. on Control, Automation, and Systems, pp. 1020-1024 (2005)

[20] M. M. Williamson, "Rhythmic Robot Arm Control Using Oscillators," Proc. IEEE/RSJ Int. Conf. on Intelligent Robots and Systems, pp.77-83. (1998)

[21] G. Taga, "A Model of the Neuro-musculo-skeletal System for Human Locomotion," Biological Cybernetics, vol. 73, pp. 97-111 (1995)

[22] O. Katayama, Y. Kurematsu, and S. Kitamura, "Theoretical Studies on Neuro Oscillator for Application of Biped Locomotion," Proc. IEEE Int. Conf. on Robotics and Automation, pp. 2871-2876 (1995) 